\title{
Congenital infantile fibrosarcoma mimicking sacrococcygeal teratoma in a Ghanaian infant: A case report and review of the literature
}

\author{
B M Duduyemi, ${ }^{1}$ MB ChB, MSc, FMC Path; A C Yifieyeh, ${ }^{2}$ MBBS, FWACS \\ ${ }^{1}$ Department of Pathology, School of Medical Sciences, Kwame Nkrumah University of Science and Technology, Kumasi, Ghana \\ ${ }^{2}$ Directorate of Surgery, Komfo Anokye Teaching Hospital, Kumasi, Ghana
}

Corresponding author: B M Duduyemi (tundeduduyemi@gmail.com)

\begin{abstract}
Congenital infantile fibrosarcoma (CIFS) is a rare tumour of childhood accounting for less than $1 \%$ of malignant tumours in children. Reports from sub-Saharan Africa are particularly rare and the occurrence in the sacral region mimicking a sacrococcygeal teratoma has not been reported in Africa to the best of our knowledge. The poor predilection of the tumour for distant metastases may contribute to its above average prognosis, but the rarity of systemic antenatal detection of congenital conditions in most parts of Africa may mitigate this good fortune. We report the very rare case of a 9-month-old female infant who presented with a progressively increasing painless sacral mass from birth which was thought to be a sacrococcygeal teratoma clinically but histopathological assessment revealed a CIFS.
\end{abstract}

S Afr J Child Health 2016;10(3):186-187. DOI:10.7196/SAJCH.2016.v10i3.955

Congenital infantile fibrosarcoma (CIFS) is a rare tumour of childhood. It accounts for $<1 \%$ of malignant tumours in children. ${ }^{[1]}$ The varied pattern of presentation of this cancer can be deduced from the occasional case reports that have emanated from almost every continent. $^{[2-5]}$ Reports from sub-Saharan Africa are particularly rare. CIFS occurrence in the sacrococcygeal region mimicking a sacrococcygeal teratoma has been reported, ${ }^{[3]}$ and such presentation is generally uncommon. The poor predilection of the tumour for distant metastases may contribute to its above-average prognosis, but the rarity of systemic antenatal detection of congenital conditions in most parts of Africa may mitigate this good fortune. ${ }^{[2,6]}$

We report the case of a 9-month-old female infant thought to have a sacrococcygeal teratoma. Pathological examination of the surgical specimen revealed a CIFS.

\section{Case report}

A 9-month-old female infant was brought to our outpatient department by a distraught mother. The infant was born with a painless sacral mass, which had been progressively increasing in size. Her mother had sought help from faith healers and traditionalists, which accounted for the late presentation. There were no associated local or systemic symptoms. There was no history of maternal illness during pregnancy nor was there use of unprescribed medications. Physical examination revealed a healthy-looking female infant who was not pale, no pedal oedema or significant lymphadenopathy. There was a $25 \times 20 \mathrm{~cm}$ mass of mixed consistency overlying the sacral region, extending to both gluteal regions and partially surrounding the anus (Fig. 1). Anal sphincteric tone was found to be slightly reduced on digital rectal examination. A tentative diagnosis of sacrococcygeal teratoma was made.

Sacral X-rays were normal. Ultrasonography confirmed the heterogeneous nature of the mass and ruled out any intra-abdominal extension. Serum alpha-fetoprotein levels were not elevated. The mass was excised via a standard Chevron incision. There was significant infiltration of both gluteal and sphincteric muscles precluding complete excision. The tip of the coccyx was excised in continuity with the mass. Grossly, we received ulcerated soft tissue measuring $12 \times 9 \times 7 \mathrm{~cm}$ covered by an eclipse of skin with cut section showing greyish-white and haemorrhagic areas. Microscopic assessment of the sacrococcygeal mass showed a hypercellular tumour composed of plump spindle cells with elongated nuclei and abundant eosinophilic cytoplasm arranged in storiform and herringbone patterns in areas. There was rare abnormal mitosis and the margins of resection were involved. These features were in keeping with CIFS (Fig. 2).

Postoperatively there was superficial surgical site infection, which was managed with povidone iodine dressings. Chemotherapy (vincristine and actinomycin D) was commenced after histopathological diagnosis. The patient's condition has been generally stable for the past 6 months and she is periodically followed up.

\section{Discussion}

CIFS has been reported as congenital in a few cases. ${ }^{[1,5]}$ In our case, the mother reported that her daughter was born with a sacral mass. The tumour is a fibroblastic soft-tissue sarcoma with a low-to-intermediate malignant potential..$^{[5,7-15]}$ An incidence of five per million infants has been reported, with males more commonly affected. ${ }^{[1,5,16]}$ CIFS can occur in any part of the body and has been found in unusual parts of the body; ${ }^{[17,18]}$ however, the extremities, namely the head, neck and trunk, in that order, are the most frequently affected locations. ${ }^{[1,4]}$ The lesions in the trunk are usually more aggressive and recurrence rates are high, especially when the excision is incomplete. Presentation in the sacral region has been reported by Al-Salem; $;{ }^{[3]}$ this is the only recorded occurrence in this location. Our patient presented in a similar fashion and we understandably made a misdiagnosis of sacrococcygeal teratoma. Sacrococcygeal teratomas present at birth as sacrococcygeal masses. ${ }^{[11]}$ Our curiosity was, however, aroused by normal serum alpha-fetoprotein levels. This tumour marker, although not diagnostic of sacrococcygeal teratomas, is elevated in many cases. ${ }^{[12]}$ The margins of resection were involved, which would have been avoided if a biopsy was taken. We admit that an atypical mass of this nature would have been biopsied for histology before definitive treatment. Because the imaging features of CIFS are non-specific, this diagnosis was not entertained following ultrasonography of the sacral mass. ${ }^{[9]}$ Infiltration of surrounding structures by the tumour is well documented. This usually limits extensive resection that would otherwise leave the patient mutilated. ${ }^{[4,15]}$ This was our experience, as both gluteal and sphincteric muscles were involved. Coccygectomy in this patient 


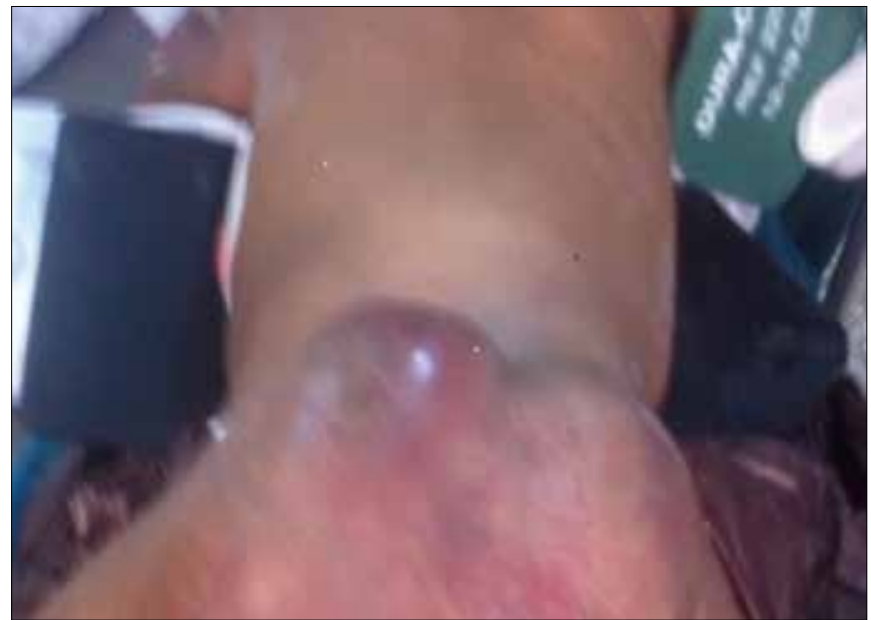

Fig. 1. A soft non-tender tissue mass on the sacrococcygeal area of the infant.

was in keeping with the general principle of reducing the risk of recurrence since we had assumed it was a sacrococcygeal teratoma. ${ }^{[13]}$

Although cytogenetic analysis of CIFS has been shown to be useful in confirming diagnosis, revealing the fusion transcript ETV6-NTRK3, such studies are not routine in our centre. The biological behaviour of the tumour and its chemosensitivity have been attributed to this defect, which itself is the result of a recurrent chromosomal translocation $\mathrm{t}(12 ; 15)\left(\mathrm{p} 13\right.$; q25). ${ }^{[1,2,5,7]}$ This is a translocation between the ETS-related transcription regulator (12p13) and tyrosine kinase cell surface receptor (15q25).

Neo-adjuvant chemotherapy is the current treatment approach, which makes surgery less mutilating. Vincristine and actinomycin $\mathrm{D}$ are the most frequently employed agents. ${ }^{[1,2,4]}$ CIFS has been successfully treated with chemotherapy alone. ${ }^{[4,5]}$ Two percent of patients in the series reported by Orbach et al. ${ }^{[4]}$ and 2 of the 11 in that by Lohl et al. ${ }^{[2]}$ received radiotherapy in addition to surgery and chemotherapy. Our patient received vincristine and actinomycin D chemotherapy. The risk of recurrence is generally high, especially in fibrosarcoma of the trunk, and therefore our patient is periodically followed up after chemotherapy. Differential diagnoses to be entertained in masses located in this abnormal area in our patient should include, but not be limited to, embryonal rhabdomyosarcoma, neurofibroma and schwannoma.

\section{Conclusion}

CIFS can mimic a sacrococcygeal teratoma. The possibility of this diagnosis should be entertained especially if the serum alphafetoprotein levels are not elevated. Radiological imaging may not help with differentiation. In our opinion, a core biopsy in such instances may help determine the appropriate therapeutic approach, as CIFS is chemosensitive. Neo-adjuvant chemotherapy would allow for complete resection without sacrificing vital structures. With the reported excellent survival rates of $>80 \%$, the outlook for our patient is good. ${ }^{[4,5]}$

\section{References}

1. Tarik E, Lamiae R, Abdelouahed A, Tarik M, Hassan G, Anouar DM. Unusual case of congenital/infantile fibrosarcoma in a new born. Afr J Paediatr Surg 2013;10(2):185-187. DOI:10.4103/0189-6725.115052

2. Loh ML, Ahn P, Perez-Atayde AR, Gebhardt MC, Shamberger RC, Grier HE. Treatment of infantile fibrosarcoma with chemotherapy and surgery: Results from the Dana-Faber Cancer Institute and Children's Hospital, Boston. J Pediatr Hematol Oncol 2002;24(9):722-726. DOI:10.1097/00043426-200212000-00008
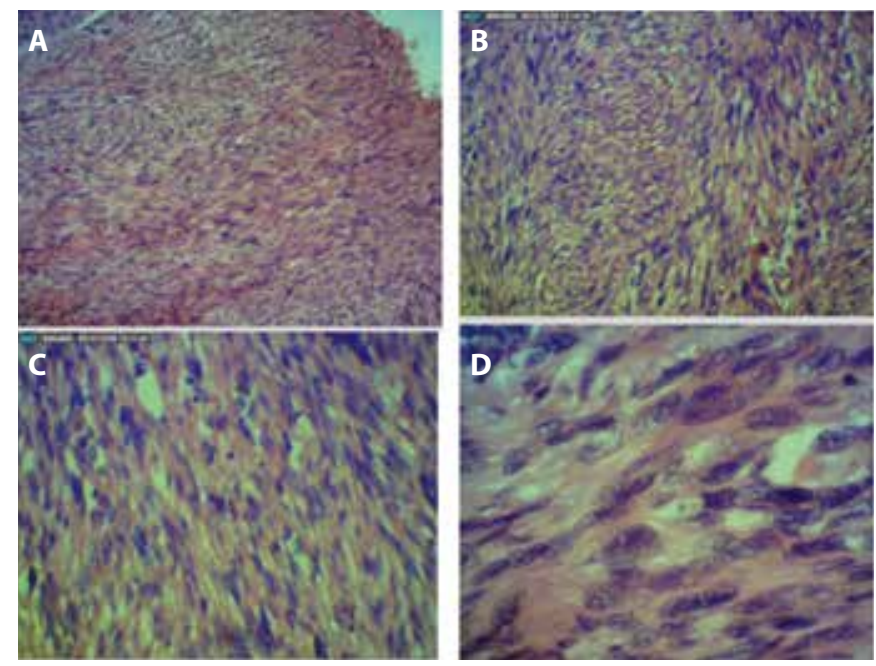

Fig. 2. Microscopy of the sacrococcygeal mass showing hypercellular tumour composed of plump spindle cells with elongated nuclei and abundant eosinophilic cytoplasm arranged in storiform and herring-bone pattern in areas. There is rare abnormal mitosis. (A, B, C and D are H\&E stains at $\times$ 40,100, 200 and 400, respectively.)

3. Al-Salem AH. Congenital infantile fibrosarcoma masquerading as sacrococcygeal teratoma. J Pediatr Surg 2011;46(11):2177-2180. DOI:10.1016/j. jpedsurg.2011.08.011

4. Orbach D, Rey A, Cecchetto G, et al. Infantile fibrosarcorma: Management based on the European experience. J Clin Oncol 2010;28(2):318-323. DOI:10.1200/JCO.2009.21.9972

5. Minard-Colin V, Orbach D, Martelli H, Bodemer C, Oberlin O. Soft tissue tumours in infants. Arch Pediatr 2009;16(7):1039-1048. DOI:10.1016/j. arcped.2009.03.005

6. Carrera JM. Obstetric ultrasounds in Africa: Is it necessary to promote their appropriate use? Donald School J Ultrasound Obstet Gynaecol 2011;5(3):289296. DOI:10.5005/jp-journals-10009-1205

7. McMahon E, Sorensen PH, Davis JH, Rogers PC, Schultz KR. Non-resectable congenital tumours with ETV6-NTRK3 gene fusion are highly responsive to chemotherapy. Med Pediatr Oncol 2003;40(5):288-292. DOI:10.1002/ mpo. 10272

8. Mukai M, Sameshima H, Kodama Y, et al. Congenital infantile fibrosarcoma in a very low-birth-weight infant. J Pediatr Surg 2012;47(4):1-4. DOI:10.1016/j. jpedsurg.2011.11.052

9. Ainsworth KE, Chavhan GB, Gupta AA, Hopyan S, Taylor G. Congenital infantile fibrosarcoma: Review of imaging features. Paediatr Radiol 2014;44(9):1124-1129. DOI:10.1007/s00247-014-2957-5

10. Akyuz C, Kupeli S, Varan A, et al. Infantile fibrosarcoma: Retrospective analysis of eleven patients. Tumori 2011;97(2):166-169. DOI:10.1700/667.7778

11. Rescorlar FJ, Sawin RS, Coran AG, Dillon PW, Azizkhan RG. Long-term outcome for infants and children with sacrococcygeal teratoma: A report from the Children's Cancer Group. J Paediatr Surg 1998;33(2):171-176.

12. Huddart SN, Mann JR, Robinson K, et al. Sacrococcygeal teratomas: The UK Children's Cancer Study Group's experience. I. Neonatal. Paediatr Surg Int 2003;19(1-2):47-51.

13. Hashish A, Fayad H, El-attar A, et al. Sacrococcygeal teratoma: Management and outcomes. Ann Paediatr Surgery 2009;5(2):119-125.

14. Kraneburg UM, Rinsky LA, Chisholm KM, Khosla RK. Emergency surgical treatment of an ulcerative and haemorrhagic congenital/infantile fibrosarcoma of the lower leg: Case report and literature review. J Pediatr Orthop B 2013;22(3):228-232. DOI:10.1097/BPB.0b013e3283536908.

15. Ferrari A, Orbach D, Sultan I, Casanova M, Bisogno G. Neonatal soft tissue sarcomas Semin Fetal Neonatal Med 2012;17(4):231-238. DOI:10.1016/j.siny.2012.05.003

16. Ries LAG, Smith MA, Gurney JG, et al. Cancer Incidence and survival Among Children and Adolescents: United States SEER Program 1975-1995, National Cancer Institute, SEER Program. NIH Pub. No. 99-4649. Bethesda, USA National Institues of Health, 1999.

17. Steelman C, Katzensteen H, Parsham D, et al. Unusual presentation of congenital infantile fibrosarcoma in seven infants with molecular genetic analysis. Fetal Pediatr Pathol 2011;30(5):329-337. DOI:10.3109/15513815.2011.587497

18. Kagon B, Shehata B, Katzensteen H, et al. Primary congenital infantile fibrosarcoma of the heart: The first confirmed case. Ann Thorac Surg 2011;91(4):166-169. DOI:10.1016/j.athoracsur.2010.08.070 